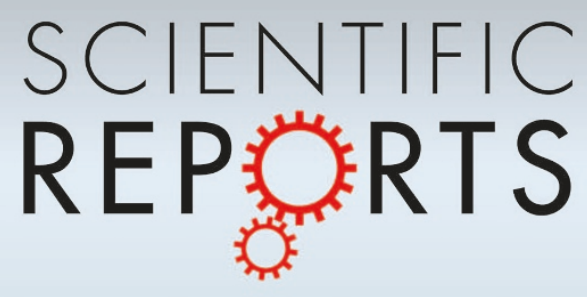

OPEN

SUBJECT AREAS:

BATTERIES

MECHANICAL ENGINEERING

COMPUTATIONAL SCIENCE

COARSE-GRAINED MODELS

Received

1 February 2013

Accepted

12 March 2013

Published

8 April 2013

Correspondence and requests for materials should be addressed to V.I.L. (vlevitas@iastate.

edu)

\section{Anisotropic Compositional Expansion and Chemical Potential for Amorphous Lithiated Silicon under Stress Tensor}

\author{
Valery I. Levitas ${ }^{1,2,3}$ \& Hamed Attariani ${ }^{1}$
}

\begin{abstract}
'lowa State University, Department of Aerospace Engineering, Ames, lowa 5001 1, U.S.A., ${ }^{2}$ lowa State University, Department of Mechanical Engineering, Ames, lowa 50011 , U.S.A., ${ }^{3}$ lowa State University, Departments of Material Science and Engineering, Ames, lowa 50011 , U.S.A.
\end{abstract}

$\mathrm{Si}$ is a promising anode material for $\mathrm{Li}$-ion batteries, since it absorbs large amounts of $\mathrm{Li}$. However, insertion of $L i$ leads to $334 \%$ of volumetric expansion, huge stresses, and fracture; it can be suppressed by utilizing nanoscale anode structures. Continuum approaches to stress relaxation in $\mathrm{Li}_{X} S \mathrm{Si}$, based on plasticity theory, are unrealistic, because the yield strength of $L i_{x} S i$ is much higher than the generated stresses. Here, we suggest that stress relaxation is due to anisotropic (tensorial) compositional straining that occurs during insertion-extraction at any deviatoric stresses. Developed theory describes known experimental and atomistic simulation data. A method to reduce stresses is predicted and confirmed by known experiments. Chemical potential has an additional contribution due to deviatoric stresses, which leads to increases in the driving force both for insertion and extraction. The results have conceptual and general character and are applicable to any material systems.

$i$ is a promising anode material for Li-ion batteries, since it absorbs large amounts of $L i^{1,2}$. Maximum insertion of $L i$ in $S i$ corresponds to $L i_{4.4} S i$, which possesses a theoretical $L i$ capacity of $4200 \mathrm{mAh} / \mathrm{g}$, an order of magnitude larger than for graphite anode ${ }^{1,2}$. However, insertion of such amount of $L i$ is accompanied by $334 \%$ percent of volumetric expansion, which under constraint conditions leads to huge stresses that may cause fracture of $L i_{x} S i$ anode ${ }^{3,4}$. This is one of the main reasons that prevents industrial application of $S i$ anodes; that is why understanding of stress development and relaxation during lithiation-delithiation is of great applied and basic importance. As we will show, this problem is also related to the development of the concept of the chemical potential under nonhydrostatic (deviatoric) stresses and large strains $s^{5-10}$. For nanoscale anodes (nanowires, particles, and films ${ }^{11-14}$ ), fracture is suppressed and large compositional volumetric deformations of $L i_{x} S i$ under constrained conditions are believed to be accommodated by plastic flow ${ }^{5,8,15,16}$. All continuum approaches to stress relaxation in $L i_{x} S i$ anodes are based on classical viscoplasticity theory ${ }^{5,8,15,16}$. However, recent atomistic calculations ${ }^{15}$ demonstrated that the yield strength of $L i_{x} S i$ is significantly higher than the generated stresses. While atomistic simulations for crystalline materials without defects (in particular, dislocations and grain boundaries) overestimate the yield strength, this should not be the case for amorphous nanomaterial. Indeed, the same atomistic calculation $\mathrm{s}^{15}$ describe well experimental data on biaxial stress relaxation in $100 \mathrm{~nm}$ thick Si film during lithiation-delithiation. This excludes plasticity as a major relaxation mechanism and makes current continuum approaches ${ }^{5,8,15,16}$ based on solely plasticity contradictory. The only qualitative concept ${ }^{15}$ that combines the chemical potential with stress intensity in the yield condition was neither justified and elaborated, nor checked against atomistic calculations or experiments.

Here, we suggested completely different approach that stress relaxation in $L i_{x} S i$ anodes occurs not due to classical plasticity when the yield condition is satisfied but due to anisotropic (tensorial) compositional straining that takes place during insertion-extraction at any deviatoric stresses. We formulated a simple rate-equation for the deviatoric part of compositional deformation rate and calibrated a single kinetic constant in it. This equation supplemented by the elasticity rule describes quantitatively well known experimental and atomistic simulation data on the biaxial stress evolution during lithiation-delithiation of $L i_{x} S i$. While here we considered the simplest model without plasticity, in some cases (e.g., for lower (fluctuating) yield strength or larger sample) combination of anisotropic compositional expansion and classical plasticity may give even better description. Since there is not any threshold stress for relaxation, we suggested a method to reduce stresses by small oscillation in Li concentration and our predictions are in qualitative agreement with known experiments. Our development in stressdependent kinematics of insertion-extraction lead to the necessity of conceptual revisiting of thermodynamics 
under general tensorial stress states and large strains. Thus, dissipation related to the insertion-extraction is introduced. Generalization of the chemical potential, $\mu$, includes an additional contribution due to deviatoric stresses, which leads to the increase in the driving force both for insertion and extraction and to prediction of unexpected phenomena. A similar approach is applicable to large compositional deformation and stress relaxation for any material systems. Also, such an approach can be applied to other processes, like chemical reactions and melting under nonhydrostatic conditions ${ }^{17,18}$, when anisotropic (tensorial) transformation strain can be introduced and described in a similar thermodynamic way.

The contraction of tensors over one and two indices is designated as $\boldsymbol{A} \cdot \boldsymbol{B}$ and $\boldsymbol{A}: \boldsymbol{B}$; superscripts -1 and $t$ denote inverse and transpose operations; subscript $s$ means symmetrization of a tensor, and I the unit tensor.

\section{Results}

The multiplicative decomposition of the deformation gradient, $\boldsymbol{F}=\boldsymbol{F}_{\boldsymbol{e}} \cdot \boldsymbol{U}_{\boldsymbol{c}}$ into elastic and compositional parts is used; $\boldsymbol{U}_{c}=\boldsymbol{U}_{c}^{t}$. This results in the decomposition of the deformation rate $\boldsymbol{d}=$ $\left(\dot{\boldsymbol{F}} \cdot \boldsymbol{F}^{-1}\right)_{s}=\left(\dot{\boldsymbol{F}}_{e} \cdot \boldsymbol{F}_{e}^{-1}\right)_{s}+\left(\boldsymbol{F}_{e} \cdot \dot{\boldsymbol{U}}_{c} \cdot \boldsymbol{U}_{c}^{-1} \cdot \boldsymbol{F}_{e}^{-1}\right)_{s}=\boldsymbol{d}_{e}+\boldsymbol{d}_{c}$ into elastic and compositional parts. Traditionally, $\boldsymbol{U}_{c}$ is considered as a spherical tensor describing isotropic volumetric expansion only. However, stresses can induce anisotropy of $L i$ and $S i$ atom distributions in order to minimize the Gibbs energy of the system and lead to tensorial $U_{c}$ (Fig. 1a).

It is convenient to present $U_{c}=J_{c}^{1 / 3} U_{c}^{S}$, where $J_{c}=\operatorname{det} \boldsymbol{U}_{c}$ characterizes ratio of elemental stress-free volumes with concentration of $L i x$ and zero, and $U_{c}^{S}$ describes isochoric change in shape. Then $\boldsymbol{d}_{c}=\dot{J}_{c} /\left(3 J_{c}\right) \boldsymbol{I}+\boldsymbol{d}_{c}^{S}$ with $\boldsymbol{d}_{c}^{S}=\left(\boldsymbol{F}_{e} \cdot \dot{\boldsymbol{U}}_{c}^{S} \cdot \boldsymbol{U}_{c}^{S-1} \cdot \boldsymbol{F}_{e}^{-1}\right)_{s}$ represents an additive decomposition of the compositional deformation rate into spherical and deviatoric parts that characterize change in volume and shape.

The initial part of the continuum thermodynamic derivations is given in the Method section. We start with the rate of dissipation per unit volume in the reference state $\Omega_{0}$ (undeformed $S i$ ) $\mathcal{D}=J \boldsymbol{\sigma}: \boldsymbol{d}-$ $\dot{\psi}+\bar{V}_{S i}^{-1} \mu \dot{x} \geq 0$, where $\rho_{0}$ is the mass density in the reference state $\Omega_{0}$, $\sigma$ is the true Cauchy stress, $\bar{V}_{S i}$ is the molar volume of $S i, \mu$ is the chemical potential of $L i$, and $J=\operatorname{det} F$ is the ratio of volumes in the deformed state of $L i_{x} S i$ in the actual configuration $\Omega$ and reference state of $S i$ in $\Omega_{0}$. Assume that the Helmholtz free energy per unit volume of $S i$ in $\Omega_{0}$ is $\psi=\psi\left(E_{e}, x\right)$ with Lagrangian elastic strain $\boldsymbol{E}_{e}=0.5\left(\boldsymbol{F}_{e}^{t} \cdot \boldsymbol{F}_{e}-\boldsymbol{I}\right)$. Then the independence of $\mathcal{D}$ of $\dot{\boldsymbol{E}}_{e}$ results in the elasticity rule (see, e.g., Ref. 19) and residual dissipation inequality

$$
\boldsymbol{\sigma}=J \boldsymbol{F}_{e} \cdot \frac{\partial \psi}{\partial \boldsymbol{E}_{e}} \cdot \boldsymbol{F}_{e}^{t} ; \quad \mathcal{D}=\left(\frac{\mu}{\bar{V}_{S i}}-\frac{\partial \psi}{\partial x}\right) \dot{x}+J \boldsymbol{\sigma}: \boldsymbol{d}_{c} \geq 0,
$$

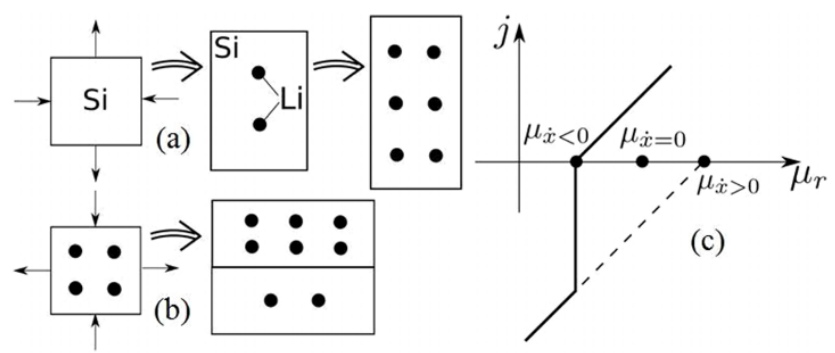

Figure $1 \mid$ (a) Anisotropic compositional deformation of $L i_{x} S i$ during lithiation under deviatoric stresses. (b) When deviatoric stress $S$, applied to a sample, produces the same driving force for insertion and extraction, $L i_{x} S i$ sample may be fluctuationally divided into two samples separated by an interface, in one of which insertion occurs and in another one extraction takes place. (c) Magnitude of $L i$ flux versus chemical potential of $L i$ reservoir $\mu_{r}$ under deviatoric stresses (solid line, Eq.(6)). which can be transformed to

$$
\mathcal{D}=\left(\frac{\mu}{\bar{V}_{S i}}-\frac{\partial \psi}{\partial x}+p_{0} J_{e} \frac{d J_{c}}{d x}\right) \dot{x}+J S: \boldsymbol{d}_{c}^{S} \geq 0,
$$

where $p_{0}$ and $S$ are the mean and deviatoric parts of the stress $\sigma$, and $J_{e}=\operatorname{det} F_{e}$ is the ratio of the volumes in the deformed and undeformed states of $L i_{x} S i\left(J=J_{e} J_{c}\right)$. If one chooses $p_{0}$ to make the parenthesis in Eq.(2) equal to zero, then inequality (2), $S: \boldsymbol{d}_{c}^{S} \geq 0$, means that $\boldsymbol{d}_{c}^{S}$ must depend on $S$, and for isotropic amorphous materials $\boldsymbol{d}_{c}^{S}$ is an isotropic function of $S$. To allow change in $\boldsymbol{U}_{c}^{S}$ during insertion-extraction only (otherwise, it would not be a compositional strain but viscous strain), one needs to impose $\boldsymbol{d}_{c}^{S}=0$ when $\dot{x}=0$. We accept the simplest expression

$$
\boldsymbol{d}_{c}^{S}=\Lambda(x) S\left|\dot{J}_{c}\right|=\Lambda(x) S \frac{d J_{c}}{d x}|\dot{x}| ; \quad \Lambda>0
$$

with the kinetic coefficient $\Lambda(x)$. We assumed that $\boldsymbol{d}_{c}^{S}$ is scaled with the magnitude of the rate of the volumetric compositional strain, $\left|\dot{J}_{c}\right|$, and $\boldsymbol{d}_{c}^{S}=0$ when $\dot{x}=0$. If we assumed that $\boldsymbol{d}_{c}^{S}$ is proportional to $\dot{x}$ rather than to $|\dot{x}|$, it would violate the dissipation inequality $S: d_{c}^{S}=\Lambda(x) S: S \frac{d J_{c}}{d x} \dot{x} \geq 0$ for $\dot{x} \leq 0$. This means that the deformation rate $\boldsymbol{d}_{c}^{S}$ is the same for insertion and extraction if $\Lambda(x)$ and $J_{c}(x)$ are the same for insertion and extraction. If $J_{c}$ is a linear function of $x$, it does not introduce extra concentration dependence; otherwise, it does. In any case, the multiplier $\frac{d J_{c}}{d x}$ can be combined with $\Lambda(x)$. A slightly better description of experimental and atomistic results from ${ }^{15}$ with $\Lambda=$ const is achieved (see below) with additional multiplier $\frac{d J_{c}}{d x}$ in Eq.(3) than without it, which is why it is separated. It should be mentioned that Eq.(3) does not describe traditional viscous relaxation and dissipation, which can be made negligible in an infinitesimally slow process. Indeed, multiplying Eq.(3) by time increment $d t$, one obtains $\left(\boldsymbol{F}_{e} \cdot d \boldsymbol{U}_{c}^{S} \cdot \boldsymbol{U}_{c}^{S-1} \cdot \boldsymbol{F}_{e}^{-1}\right)_{s}=\Lambda(x) S\left|d J_{c}\right|$, i.e., relaxation Eq.(3) is time independent, and the magnitude of the volumetric strain or concentration $|x|$ plays a role of a time-like parameter. This is similar to the flow rule in classical elastoplasticity, which is time-independent, and plastic strain plays a part of a time-like parameter. Thus, even for infinitesimally slow lithiationdelithiation, the problem is thermodynamically nonequilibrium, history-dependent, and cannot be reduced to minimization of the free energy functional.

Traditionally (for $\boldsymbol{d}_{c}^{S}=0$ ), the compositional dissipation rate is assumed to be zero, which defines an expression for the chemical potential $\mu$. In our more general case with $\boldsymbol{d}_{c}^{S} \neq 0$, we postulate

$$
\mathcal{D}=\zeta J \boldsymbol{S}: \boldsymbol{d}_{c}^{S}=\zeta \Lambda J \boldsymbol{S}: S \frac{d J_{c}}{d x} \operatorname{sign}(\dot{x}) \dot{x} \geq 0
$$

with the parameter $\zeta(0 \leq \zeta \leq 1)$ and $|\dot{x}|=\dot{x} \operatorname{sign}(\dot{x})$ (assuming $\operatorname{sign}(0)$ $=0)$. Then the remaining part of Eq.(2) is zero, which defines the generalized chemical potential

$$
\frac{\mu}{\bar{V}_{S i}}=\frac{\partial \psi}{\partial x}-p_{0} J_{e} \frac{d J_{c}}{d x}-\Lambda(1-\zeta) J S: S \frac{d J_{c}}{d x} \operatorname{sign}(\dot{x}) .
$$

Let us consider a $L i$ reservoir with a chemical potential $\mu_{r}$ in contact with a small $L i_{x} S i$ sample (i.e., heterogeneities of all parameters can be neglected) with the chemical potential of $L i \mu$. Let the magnitude of the flux of $L i$ to the sample in the reference state be described by experimentally determined function $j=b \bar{V}_{S i}^{-1} x \Delta \mu / \Delta y$, with the size $\Delta y$ of the order of magnitude of the size of a $L i_{x} S i$ sample, $\Delta \mu=\mu_{r}-$ $\mu$, and with mobility coefficient $b$. An unusual feature of Eq.(5) is that the last term due to the deviatoric stresses always increases the magnitude of the driving force, $|\Delta \mu|$, for the $L i$ transport both for insertion and extraction, i.e., it decreases $\mu$ for insertion and increases $\mu$ 
for the extraction. This is, however, logical, because if $\boldsymbol{S}$ represents internal stresses that appear due to volumetric change during insertion or extraction and suppress these processes, then Eq.(3) describes the relaxation of internal stresses, which should increase $|\Delta \mu|$. If $S$ represents prescribed external stresses, then they produce positive compositional work along the $\boldsymbol{d}_{c}^{S}$ that also increases $|\Delta \mu|$.

This, however, leads to an unusual situation for small driving forces $|\Delta \mu|$, when the driving force is positive simultaneously for both insertion and extraction. Indeed, let the under hydrostatic condition $L i_{x} S i$ sample be in thermodynamic equilibrium with the reservoir, i.e., $\mu_{r}=\mu_{\dot{x}=0}$. For simplicity shear modulus is independent of $x$. If we apply deviatoric stress $S$ to the sample, according to Eq.(5), chemical potential of $L i$ in $L i_{x} S i$ will be reduced for $\dot{x}>0$ and increased for $\dot{x}<0$ by the same value, i.e., deviatoric stress produces the same driving force for insertion and extraction. Let under applied deviatoric stresses, a $L i_{x} S i$ sample be fluctuationally divided into two samples separated by an interface, and in one of them insertion occurred and in another one extraction took place (Fig. 1b). We neglect interface energy and elastic energy due to a jump in compositional transformation strain across an interface. However, chemical potential of a sample with $\dot{x}>0$ is smaller than that for a sample with $\dot{x}<0$, i.e., $\mu_{\dot{x}>0}<\mu_{\dot{x}<0}$, and the chemical potential of such a heterogeneous sample is larger than the potential $\mu_{\dot{x}>0}$ when the entire sample undergoes insertion. Thus, it is more probable that the system evolves in the way minimizing the chemical potential. Consequently (Fig. 1c),

$$
\begin{aligned}
& \text { if } \mu_{r}>\mu_{\dot{x}>0} \rightarrow \dot{x}>0 \text { and } j=b \bar{V}_{S i}^{-1} x\left(\mu_{r}-\mu_{\dot{x}>0}\right) / \Delta y \text {; } \\
& \text { if } \mu_{r} \leq \mu_{\dot{x}>0} \rightarrow \dot{x}<0 \text { and } j=b \bar{V}_{S i}^{-1} x\left(\mu_{r}-\mu_{\dot{x}<0}\right) / \Delta y .
\end{aligned}
$$

Eq.(6) means that if the process leading to the minimal chemical potential (i.e., insertion) can occur, it will occur, despite the fact that the opposite process could occur faster for $\mu_{\dot{x}>0}<\mu_{r}<\mu_{\dot{x}=0}$, but leads to higher chemical potential. Also, for $\mu_{\dot{x}>0}=\mu_{r}$, when only extraction is possible, there is a jump in flux, because $\mu_{r}-\mu_{\dot{x}<0}$ is finite. This jump and corresponding jump in the chemical potential, $\mu_{\dot{x}<0}-\mu_{\dot{x}>0}$, can be used for experimental determination of the parameter $\zeta$.

In all atomistic simulations (see, e.g., Ref. 20), elastic moduli are calculated by considering elastic perturbation taking unloaded configuration $\Omega_{c}$ (i.e., at $F_{e}=I$ ) as the reference one. It automatically assumes independence of the elasticity rule of $\boldsymbol{U}_{c}$ and the reference configuration. We accept the simplest expression for the free energy per unit volume of the unloaded configuration $\Omega_{c}, J_{c}^{-1} \psi=$ $\psi^{c}+0.5 \boldsymbol{E}_{e}: \boldsymbol{C}(x): \boldsymbol{E}_{e}$, where $\psi^{c}$ is the concentrational part of the free energy per unit volume in $\Omega_{c}$ and $C$ is the forth-rank tensor of isotropic elastic moduli. Then the elasticity rule Eq.(1) is

$$
\boldsymbol{\sigma}=J_{e}^{-1} \boldsymbol{F}_{e} \cdot\left(\boldsymbol{C}(x): \boldsymbol{E}_{e}\right) \cdot \boldsymbol{F}_{e}^{t} ; \quad J_{e}=\operatorname{det} \boldsymbol{F}_{e} .
$$

Note that in Ref. 8 the same expression for the elastic energy is accepted, but in the reference configuration, which leads to the equation $\boldsymbol{\sigma}=J_{e}^{-1} J_{c}^{-1} \boldsymbol{F}_{e} \cdot\left(\boldsymbol{C}: \boldsymbol{E}_{e}\right) \cdot \boldsymbol{F}_{e}^{t}$. It differs from Eq.(7) by a factor of $J_{c}^{-1}$, which may be smaller than $1 / 4$ because of large compositional volume change. Thus, if elasticity moduli are determined like in Ref. 20 in the unloaded configuration, utilization of the reference configuration for the elasticity rule introduces strong nonphysical dependence of the elasticity rule on compositional volume change $J_{c}^{-1}$ and corresponding nonphysical contribution to the chemical potential. Note that in Ref. 8 such a discrepancy between different definitions of elastic moduli was mentioned and to some extent was taken into account by accepting that their Young's modulus is an increasing function of $x$ (while the Young's modulus in Ref. 20 is a decreasing function of $x$ ).

Eqs.(3), (7), and $F=F_{e} \cdot U_{c}$ have been applied to the problem of the biaxial stress generation and relaxation in $\mathrm{Si}$ film-like anode during lithiation-delithiation on the rigid substrate in the formulation similar to that in Ref. 8,15. Diffusion is considered to be fast enough so that homogeneous $x$ and all fields are assumed. The boundary conditions are for in-plane principal components of the deformation gradient $F_{1}=F_{2}=1$ and for out-of-plane principle stress component $\sigma_{3}=0$. The following approximations for $J_{c}=$ $1+\varepsilon_{0}$ with $\varepsilon_{0}^{l}=0.01\left(12.37 x^{4}-58.72 x^{3}+100 x^{2}+3.326 x\right)$ for lithiation and $\varepsilon_{0}^{d l}=0.01\left(1.037 x^{4}-12.97 x^{3}+31.04 x^{2}+44.27 x+9.187\right)$ for delithiation, and for biaxial elastic modulus $E /(1-v)=$ 127.1exp $(-0.5576 x)$ Gpa describe well experimental data and atomistic simulations in Ref. 20,21, where $E$ and $v$ are the Young's modulus and Poisson's ratio. The only unknown constant $\Lambda=$ $0.47 \mathrm{GPa}^{-1}$ is chosen for the best fit of biaxial stress $\sigma_{1}=\sigma_{2}$ at $x=$ 2 to experiment and atomistic simulations in Ref. 15. Fig. 2 demonstrates remarkable qualitative and quantitative agreements of our results for evolution $\sigma=\sigma_{1}(x)$ for lithiation-delithiation with experiment and atomistic simulations in Ref. 15. We could get even better agreement, if necessary, by proper fitting for $\Lambda(x)$ and using different $\Lambda(x)$ for lithiation and delithiation, but the main point is that agreement is good enough with just one chosen material coefficient $\Lambda$. In comparison, the viscoplastic model in Ref. 8 fits two material constants and one function (the yield strength) of $x$ and still does not have such a good quantitative correspondence with experiments. The main reason is that the model in Ref. 8 allows for stress relaxation above the yield strength only. This underscores the importance of a conceptually correct model for stress relaxation.

\section{Discussion}

Since in our model there is not a threshold for the stress relaxation and it occurs both for lithiation-delithiation, one can suggest a simple method to reduce internal stresses by applying oscillatory change in $x$ with a small magnitude, e.g., $x=a+a \sin (2 \pi \tilde{t})$; here $\tilde{t}=t / 100 s$ is the dimensionless time and $100 \mathrm{~s}$ is a typical diffusion time for lithium through a $100 \mathrm{~nm}$ silicon film ${ }^{15}$. In Fig. 3a, oscillations started after almost completion of delithiation in Fig. 2, in order to completely release stresses before some pause in the battery operation. Such a significant reduction in stresses is in qualitative agreement with experiments $^{22}$. In contrast, simulation of cyclic lithiation-delithiation in Ref. 8 results in almost constant stresses. In Fig. 3b, we complete the task to keep tensile stresses below $1 G P a$ during delithiation by superposition of multiple oscillatory change in $x, x=x_{0}+$ $0.01 \sin (2 \pi \tilde{t})$ when stress reaches $1 \mathrm{GPa}$ until it reduces to zero.

To summarize, a mechanism of stress relaxation during insertionextraction based on the anisotropic (tensorial) compositional strain is suggested and described theoretically. Theoretical results and the suggested method to reduce stresses are in good correspondence with experiments. The general concepts of the chemical potential and

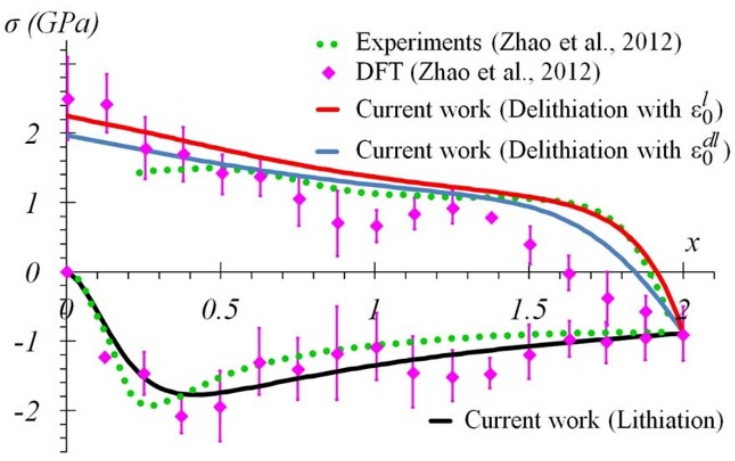

Figure $2 \mid$ Calculated biaxial stress during lithiation-delithiation is in remarkable good correspondence with experimental results and density functional theory (DFT) simulations from ${ }^{15}$, with just one fitted material parameter $\Lambda$. For delithiation, two options were used: with volumetric strain $\varepsilon_{0}^{d l}$ and with the same $\varepsilon_{0}^{l}$ as for lithiation. 


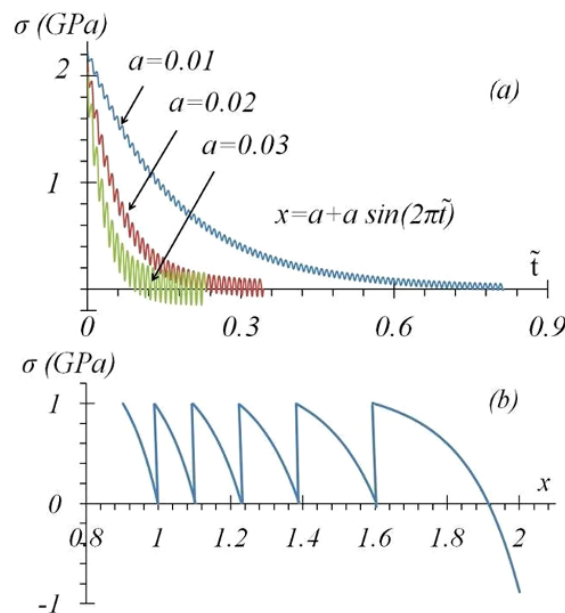

Figure 3 (a) Reduction in biaxial stress with time after almost completion of delithiation ( $x=a$ in Fig. 2) under oscillatory change in $x$. (b) Keeping tensile stresses below $1 \mathrm{GPa}$ during delithiation by superposition of multiple oscillatory change in $x, x=x_{0}+0.01 \sin (2 \pi \tilde{t})$ when stress reaches $1 \mathrm{GPa}$ until it reduces to zero (vertical lines).

dissipation due to insertion-extraction are advanced for an arbitrary material system.

\section{Methods}

The large strain continuum thermodynamic approach under general nonhydrostatic conditions is utilized. Kinematics of large deformations with multiple intermediate configurations is described in Ref. 19. Here, kinematics is based on the multiplicative decomposition of the deformation gradient into elastic and compositional parts. Compositional part includes change in volume and shape, i.e., it represents a secondrank tensor. Plastic deformation is neglected because stresses are below the yield strength. We accepted the expression for the free energy per unit volume of the unloaded configuration, which allowed us to directly use results of atomistic simulations for elastic moduli. The initial part of the continuum thermodynamic derivations is similar to that in Ref. 7-10. However, we introduce the dissipation rate due to insertion and extraction. An additional term appears in the dissipation rate due to tensorial compositional strain. Some detail are described below and in the main text.

Three configurations will be considered: the reference configuration $\Omega_{0}$, which corresponds to the undeformed $S i$ state; actual configuration $\Omega$, corresponding to the actual deformed state of $L i_{x} S i$ and unloaded configuration $\Omega_{c}$, obtained after release of external stresses after deformation of $L i_{x} S i$ without change in $x$. All derivations will be performed in the reference configuration $\Omega_{0}$. For the molar fraction of $L i$ per mole of $S i, x$, the following mass balance equation is valid in $\Omega_{0}$

$$
\dot{x}+\bar{V}_{S i} \nabla \cdot \boldsymbol{j}=0,
$$

where $\bar{V}_{S i}$ is the molar volume of $S i$ and $j$ is the flux of the $L i$ defined as a number of moles per unit reference area per unit time; $\nabla$ and $\nabla \cdot$ are the gradient and divergence operators in $\Omega_{0}$, respectively. The first and second laws of thermodynamics for the reference volume $V_{0}$ for neglected volumetric forces, heat supplies and kinetic energy are:

$$
\begin{gathered}
\int_{S_{0}}(\boldsymbol{v} \cdot \boldsymbol{P} \cdot \boldsymbol{n}-\boldsymbol{h} \cdot \boldsymbol{n}-\mu \boldsymbol{j} \cdot \boldsymbol{n}) d S_{0}-\frac{d}{d t} \int_{V_{0}} U d V_{0}=0, \\
\frac{d}{d t} \int_{V_{0}} s d V_{0}+\int_{S_{0}} \frac{\boldsymbol{h}}{\theta} \cdot \boldsymbol{n} d S_{0} \geq 0 .
\end{gathered}
$$

Here $\boldsymbol{v}$ is the material velocity, $\boldsymbol{h}$ the heat flux, $\boldsymbol{n}$ is the unit normal to the external surface $S_{0}, U$ is the specific (per unit volume of $S i$ ) internal energy, $s$ is the specific entropy, $\theta$ is the temperature, $\mu$ is the chemical potential of $L i$, and $\boldsymbol{P}$ is the first nonsymmetric Piola-Kirchoff (nominal) stress tensor (the force per unit area in the undeformed state). Using the Green-Gauss theorem to transform surface to volume integrals, $\nabla \boldsymbol{v}=\dot{\boldsymbol{F}}$, as well as the equilibrium equation $\nabla \cdot \boldsymbol{P}=0$, we transform Eqs.(9) and (10) to volumetric integrals and then to the local form

$$
\begin{gathered}
\boldsymbol{P}^{t}: \dot{\boldsymbol{F}}-\dot{U}-\nabla \cdot \boldsymbol{h}-\mu \nabla \cdot \boldsymbol{j}-\boldsymbol{j} \cdot \nabla \mu=0, \\
\dot{\boldsymbol{s}}+\frac{1}{\theta} \nabla \cdot \boldsymbol{h}-\frac{\nabla \theta}{\theta^{2}} \cdot \boldsymbol{h} \geq 0 .
\end{gathered}
$$

Combining Eq.(11) and Eq.(12) multiplied by the temperature, we obtain the dissipation inequality

$$
\boldsymbol{P}^{t}: \dot{\boldsymbol{F}}-\dot{U}+\theta \dot{\boldsymbol{s}}-\frac{\nabla \theta}{\theta} \cdot \boldsymbol{h}-\mu \nabla \cdot \boldsymbol{j}-\boldsymbol{j} \cdot \nabla \mu \geq 0
$$

Introducing the Helmholtz free energy per unit volume of $S i$ in $\Omega_{0}, \psi=U-\theta s$; utilizing the balance Eq.(8) and stress power identity $\boldsymbol{P}^{t}: \dot{\boldsymbol{F}}=J \boldsymbol{\sigma}: \boldsymbol{d}$ (see, e.g., Ref. 19), with the symmetric true Cauchy stress $\sigma$, deformation rate $\boldsymbol{d}$, and the volume ratio in the actual and reference configurations $J=\operatorname{det} \boldsymbol{F}=d V / d V_{0}$, as well as substituting inequality (13) with three stronger inequalities, we obtain

$$
\mathcal{D}=J \boldsymbol{\sigma}: \boldsymbol{d}-\dot{\psi}-s \dot{\theta}+\bar{V}_{S i}^{-1} \mu \dot{x} \geq 0 ; \quad-\frac{\nabla \theta}{\theta} \cdot \boldsymbol{h} \geq 0 ; \quad-j \cdot \nabla \mu \geq 0 .
$$

Here we introduced the dissipation rate per unit volume in $\Omega_{0}, \mathcal{D}$. The isothermal version of the dissipation inequality $\mathcal{D} \geq 0$ is the initial point in the main text.

1. Tarascon, J. M. \& Armand, M. Issues and challenges facing rechargeable lithium batteries. Nature 414, 359-367 (2001).

2. Sethuraman, V. A., Hardwick, L. J., Srinivasan, V. \& Kostecki, R. Surface structural disordering in graphite upon lithium intercalation/deintercalation. J. Power Sources 195, 3655-3660 (2010).

3. Hu, Y. H., Zhao, X. H. \& Suo, Z. G. Averting cracks caused by insertion reaction in lithium-ion batteries. J. Mater. Res. 25, 1007-1010 (2010).

4. Bhandakkar, T. K. \& Gao, H. J. Cohesive modeling of crack nucleation under diffusion induced stresses in a thin strip: Implications on the critical size for flaw tolerant battery electrodes. Int. J. Solids Struct. 47, 1424-34 (2010).

5. Cui, Z., Gao, Z. \& Qu, J. A finite deformation stress-dependent chemical potential and its applications to lithium ion batteries. J. Mech. Phys. Solids 60, 1280-1295 (2012).

6. Grinfeld, M. A. Thermodynamic Methods in the Theory of Heterogeneous Systems. (Sussex, Longman, 1991).

7. Larche, F. \& Cahn, J. W. Non-linear theory of thermochemical equilibrium of solids under stress. Acta Metall. 26, 53-60 (1978).

8. Bower, A. F., Guduru, P. R. \& Sethuraman, V. A. A finite strain model of stress, diffusion, plastic flow, and electrochemical reactions in a lithiumion half cell. J. Mech. Phys. Solids 59, 804-828 (2011).

9. Levitas, V. I. Structural changes without stable intermediate state in inelastic material. Int. J. Plasticity 16, 805-849 and 851-892 (2000).

10. $\mathrm{Wu}, \mathrm{C} . \mathrm{H}$. The role of Eshelby stress in composition-generated and stress-assisted diffusion. J. Mech. Phys. Solids 49, 1771-1794 (2001).

11. Arico, A. S., Bruce, P., Scrosati, B., Tarascon, J. M. \& Schalkwijk, W. V. Nanostructured materials for advanced energy conversion and storage devices. Nature 4, 366-377 (2005).

12. $\mathrm{Wu}, \mathrm{H}$. et al. Stable cycling of double-walled silicon nanotube battery anodes through solid electrolyte interphase control. Nat. Nanotechnol. 7, 310-315 (2012).

13. Chan, C. K. et al. High-performance lithium battery anodes using silicon nanowires. Nat. Nanotechnol. 3, 31-35 (2008).

14. Liu, X. H. et al. In situ atomic-scale imaging of electrochemical lithiation in silicon. Nat. Nanotechnol. 7, 749-756 (2012).

15. Zhao, K. et al. Reactive flow in silicon electrodes assisted by the insertion of lithium. Nano Lett. 12, 4397-4403 (2012).

16. Zhao, K., Pharr, M., Cai, S., Vlassak, J. J. \& Suo, Z. Large plastic deformation in high-capacity lithium ion batteries caused by charge and discharge. J. Am. Ceram. Soc. 94, S226-S235 (2011).

17. Levitas, V. I. \& Samani, K. Size and mechanics effects in surface-induced melting of nanoparticles. Nat. Commun. 2, 1-6 (2011).

18. Levitas, V. I. \& Samani, K. Coherent solid/liquid interface with stress relaxation in a phase-field approach to the melting/solidification transition. Phys. Rev. B. 84, 140103(R) (2011).

19. Levitas, V. I. Large Deformation of Materials with Complex Rheological Properties at Normal and High Pressure. (New York, Nova Science Publishers, 1996).

20. Shenoy, V. B., Johari, P. \& Qi, Y. Elastic softening of amorphous and crystalline Li-Si phases with increasing Li concentration: A first-principles study. J. Power Sources 195, 6825-6830 (2010).

21. Sethuraman, V. A., Chon, M. J., Shimshak, M., Van Winkle, N. \& Guduru, P. R. In situ measurement of biaxial modulus of $\mathrm{Si}$ anode for Li-ion batteries. Electrochem. Commun. 12, 1614-1617 (2010).

22. Sethuraman, V., Srinivasan, A. F. \& Bower \& Guduru, P. R. In situ measurements of stress-potential coupling in lithiated silicon. J. Electrochem. Soc. 157, A1253A1261 (2010).

\section{Acknowledgements}

This work was supported by NSF, ONR, and ISU.

\section{Author contributions}

V.I.L. proposed the research and developed theory. H.A. performed the simulations. 


\section{Additional information}

Competing financial interests: The authors declare no competing financial interests. License: This work is licensed under a Creative Commons

Attribution-NonCommercial-NoDerivs 3.0 Unported License. To view a copy of this license, visit http://creativecommons.org/licenses/by-nc-nd/3.0/
How to cite this article: Levitas, V.I. \& Attariani, H. Anisotropic Compositional Expansion and Chemical Potential for Amorphous Lithiated Silicon under Stress Tensor. Sci. Rep. 3, 1615; DOI:10.1038/srep01615 (2013). 